\title{
Fermilab
}

\section{A conduction-cooled SRF cavity: Apparatus and first results}

R. C. Dhuley ${ }^{1}$, M. I. Geelhoed ${ }^{1}$, Y. Zhao ${ }^{2}$, I. Terechkine ${ }^{1}$, M. Alvarez ${ }^{1}$, O. Prokofiev ${ }^{1}$, J. C. T. Thangaraj ${ }^{1}$

${ }^{1}$ Fermi National Accelerator Laboratory, Batavia, Illinois 2Euclid Techlabs LLC, Bolingbrook, Illinois

2019 Cryogenic Engineering Conference, Hartford, Connecticut 


\section{Goal: To demonstrate cryogen-free SRF cavity operation}

Take out liquid helium (and its complexities)

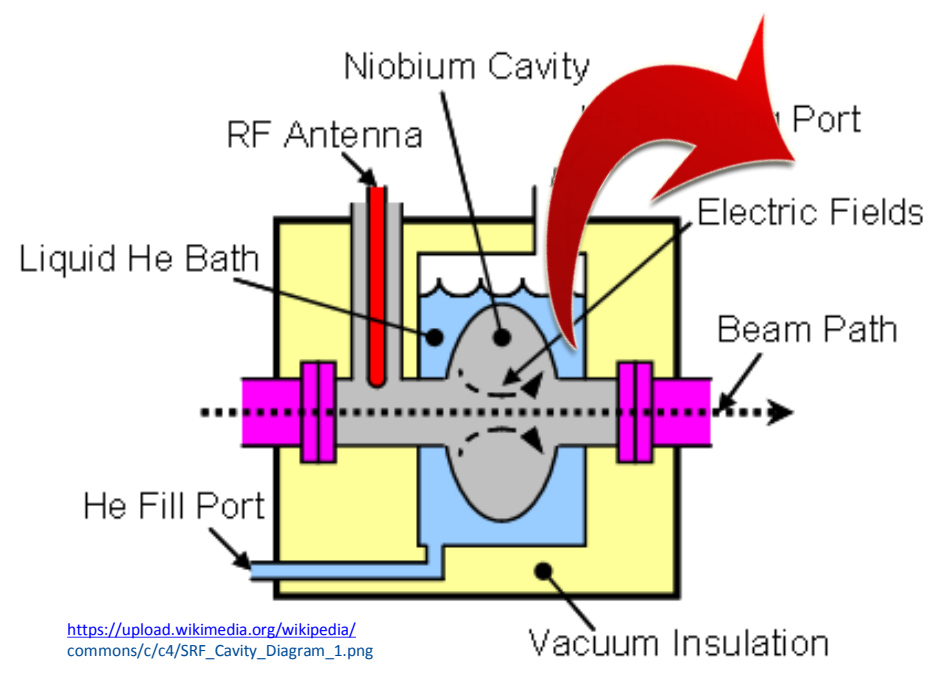

Cool SRF cavities conductively with $4 \mathrm{~K}$ cryocoolers

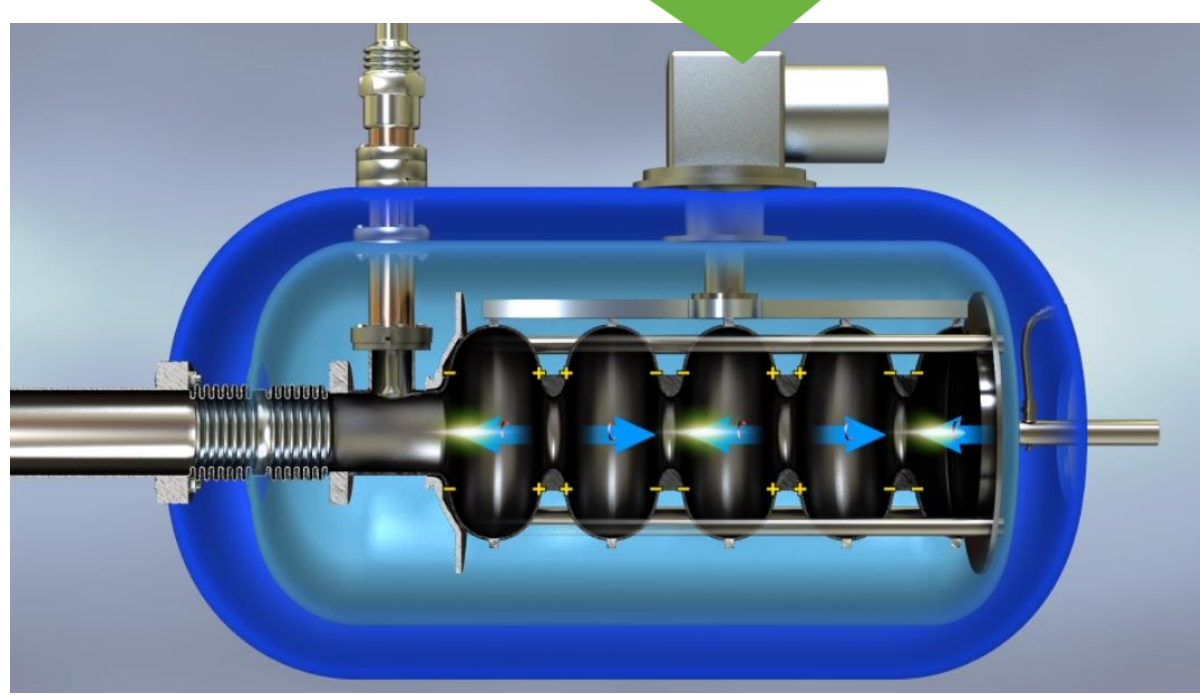

Key thermal design criterion

- SRF cavities dissipate heat during operation (dynamic heat load)

- Cryocoolers have limited $4 \mathrm{~K}$ cooling capacity

- Need a high thermal conductance link to extract this dynamic load and transport to the cryocooler. 


\section{Cavity-cooler thermal link: Our design approach}

Surface magnetic fields dissipate most heat near the equator

$$
P_{\text {diss }}=\frac{1}{2} R_{s} f\left|H_{s}\right|^{2} d s
$$

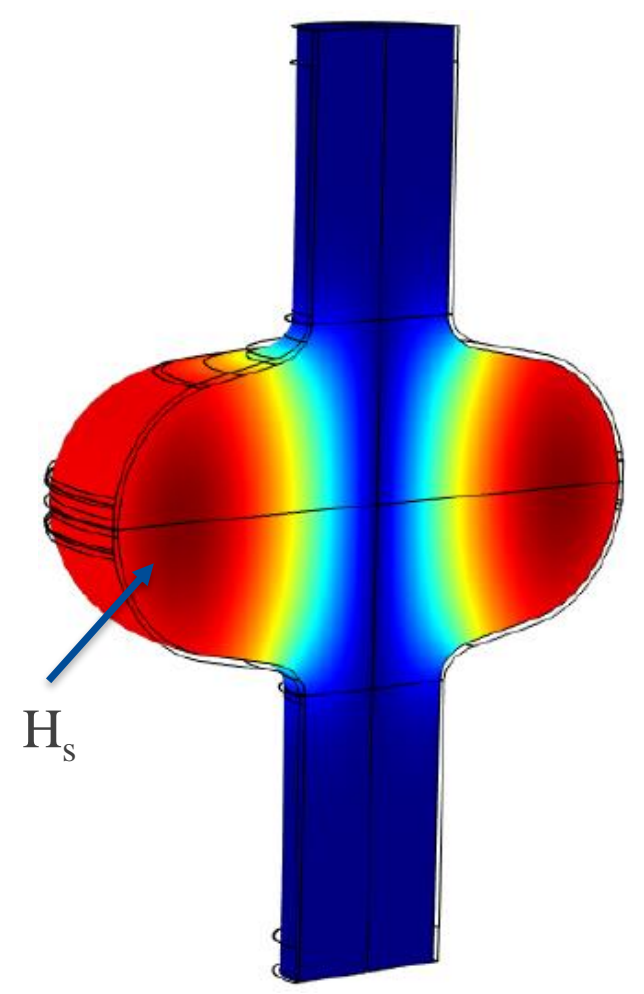

E-beam weld niobium rings around the equator to attach a thermal link

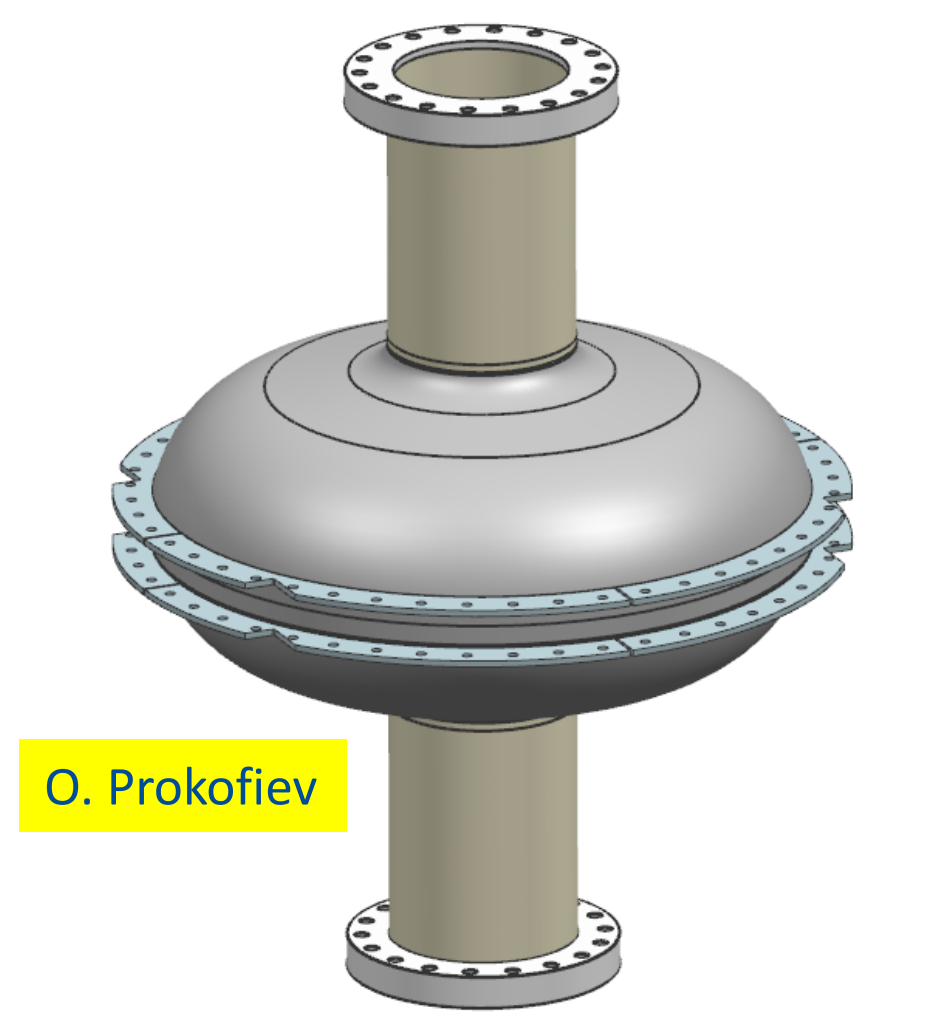




\section{Cavity-cooler thermal link: Our design approach}

Use high purity ( $5 N$ ) aluminum as the thermal link material

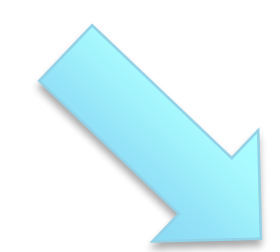

Measure and design low thermal resistance pressed niobium-aluminum contacts

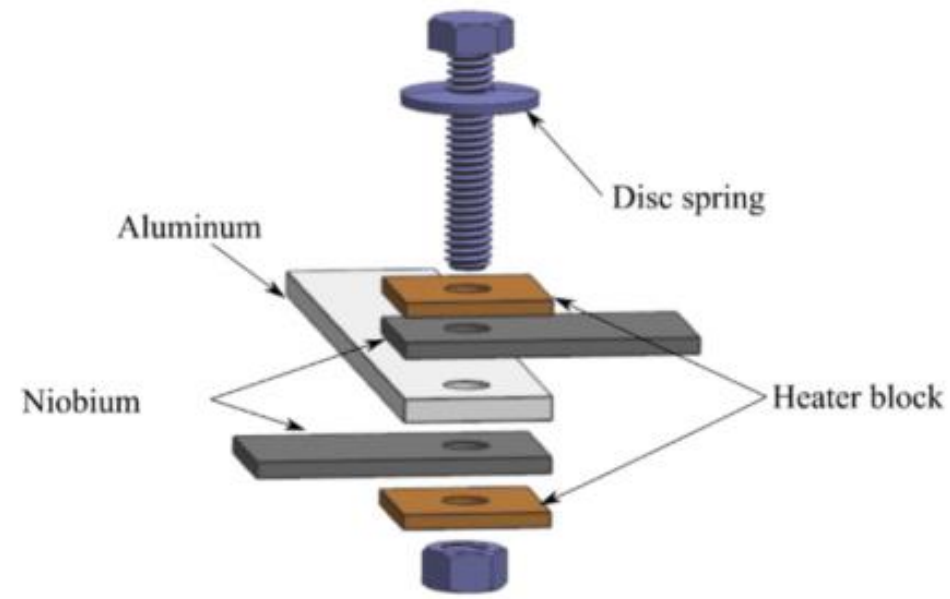

R. C. Dhuley et al., Cryogenics 93, 86-93, 2018
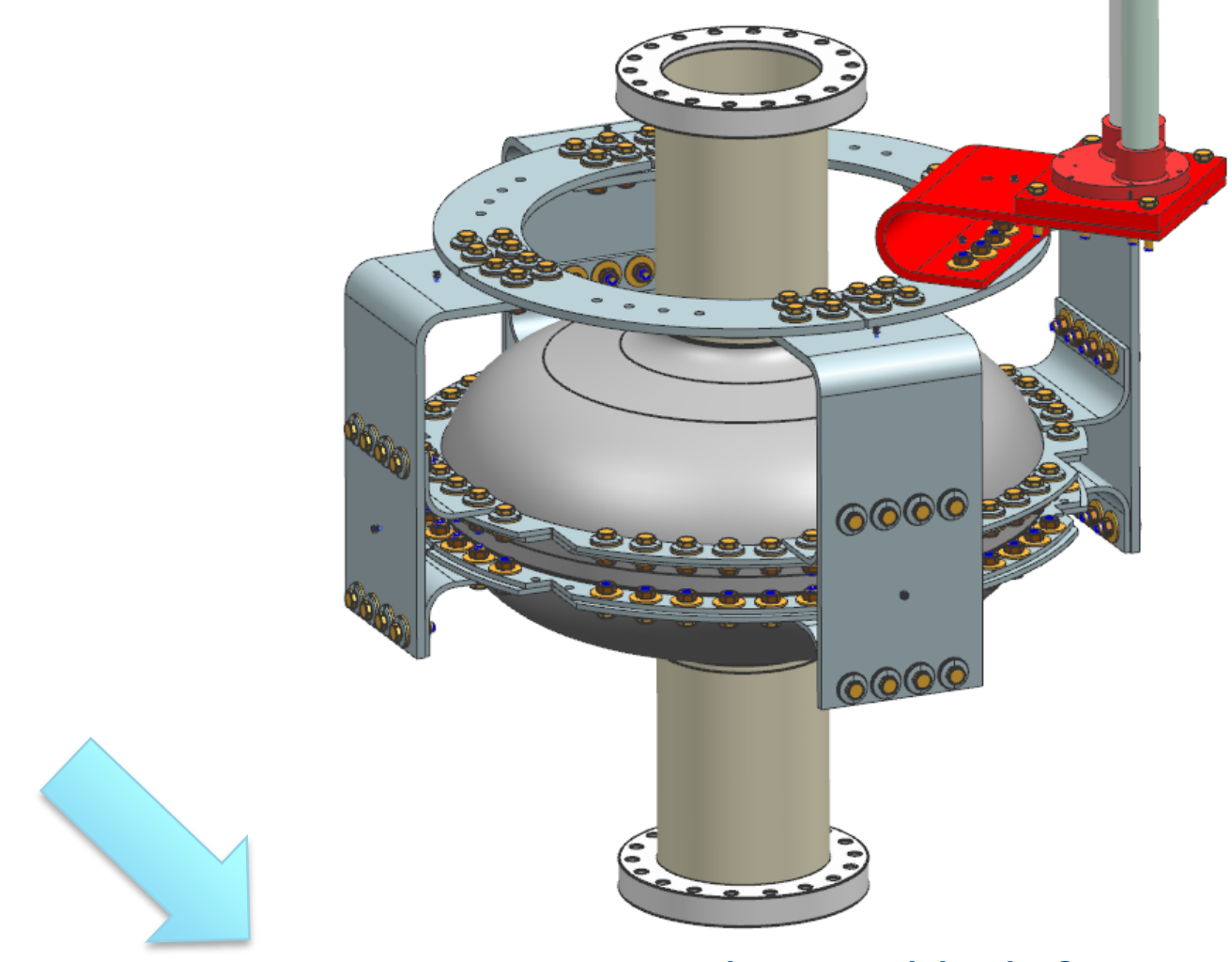

Construct a thermal link for distributed cooling around the cavity equator

R. C. Dhuley et al., IEEE TAS 29(5), 0500205, 2019 荤 Fermilab 


\section{Conduction cooled cavity test setup}

Vacuum vessel

- SS304

- 5 feet tall

M. Alvarez

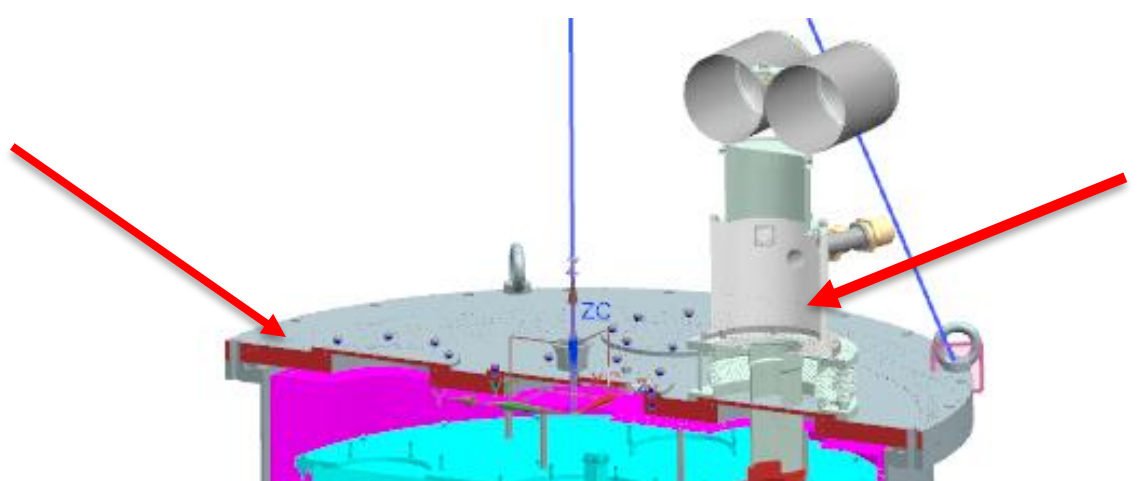

Cryocooler

- Cryomech PT420 (2 W@ @ 4.2 K with 55 W @ 45 K)

Magnetic shield

- MuMetal

- Room temperature

- $\quad<10 \mathrm{mG}$ total field at the cavity location

I. Terechkine

\section{MLI wrapped} thermal shield

- Cooled by cryocooler stage-1

- Copper 101 top plate

- Aluminum 1100 shell

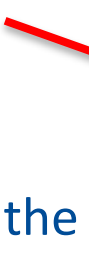

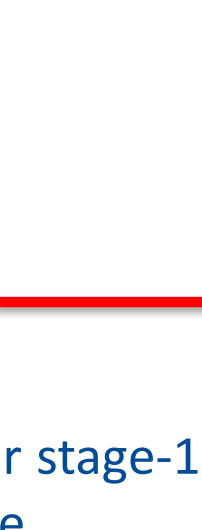




\section{Conduction cooled cavity test setup}

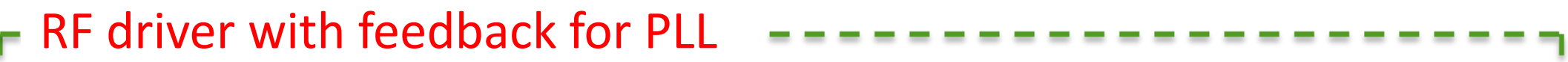

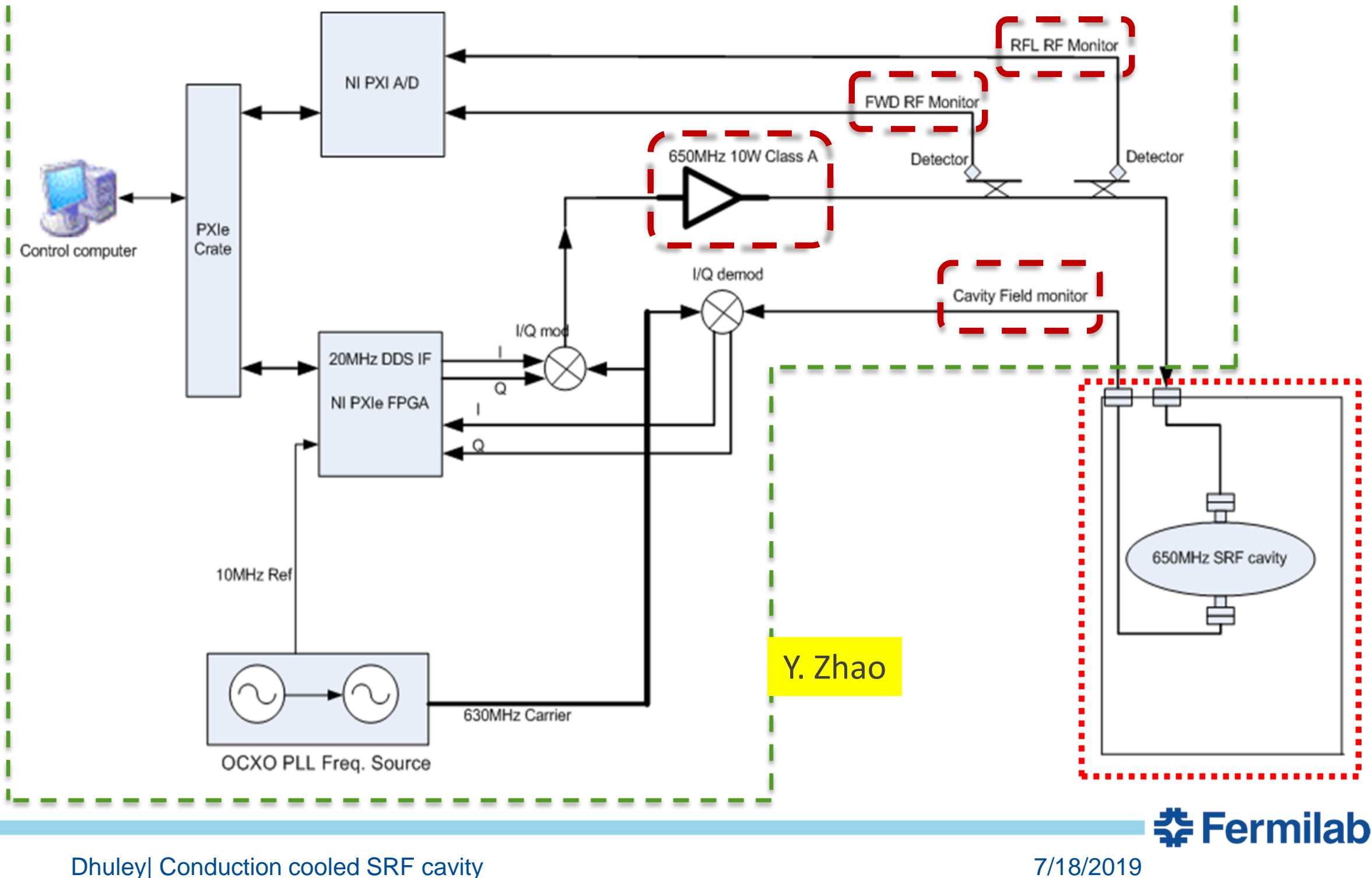




\section{Conduction cooled cavity test setup}

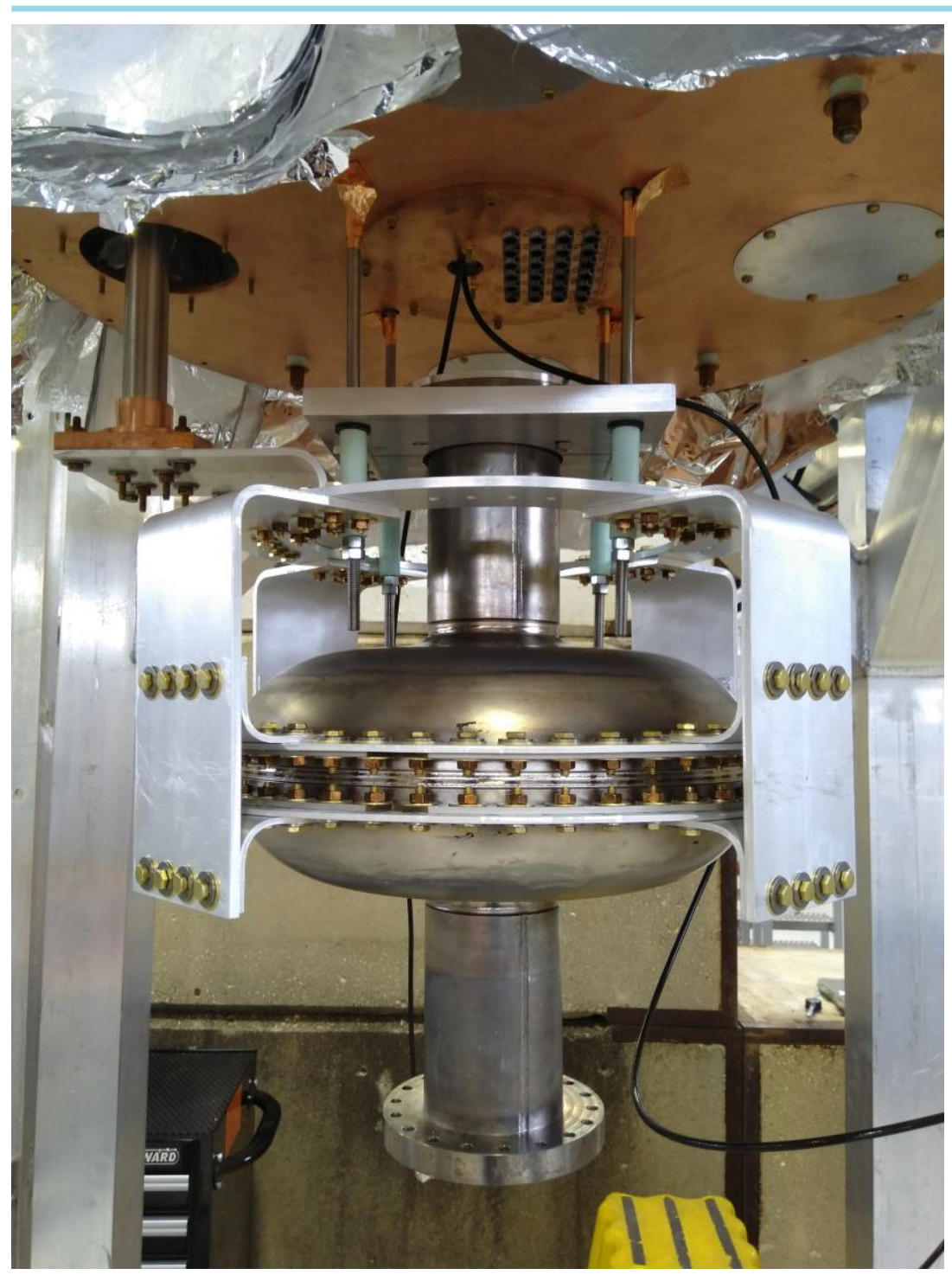

Dhuley| Conduction cooled SRF cavity

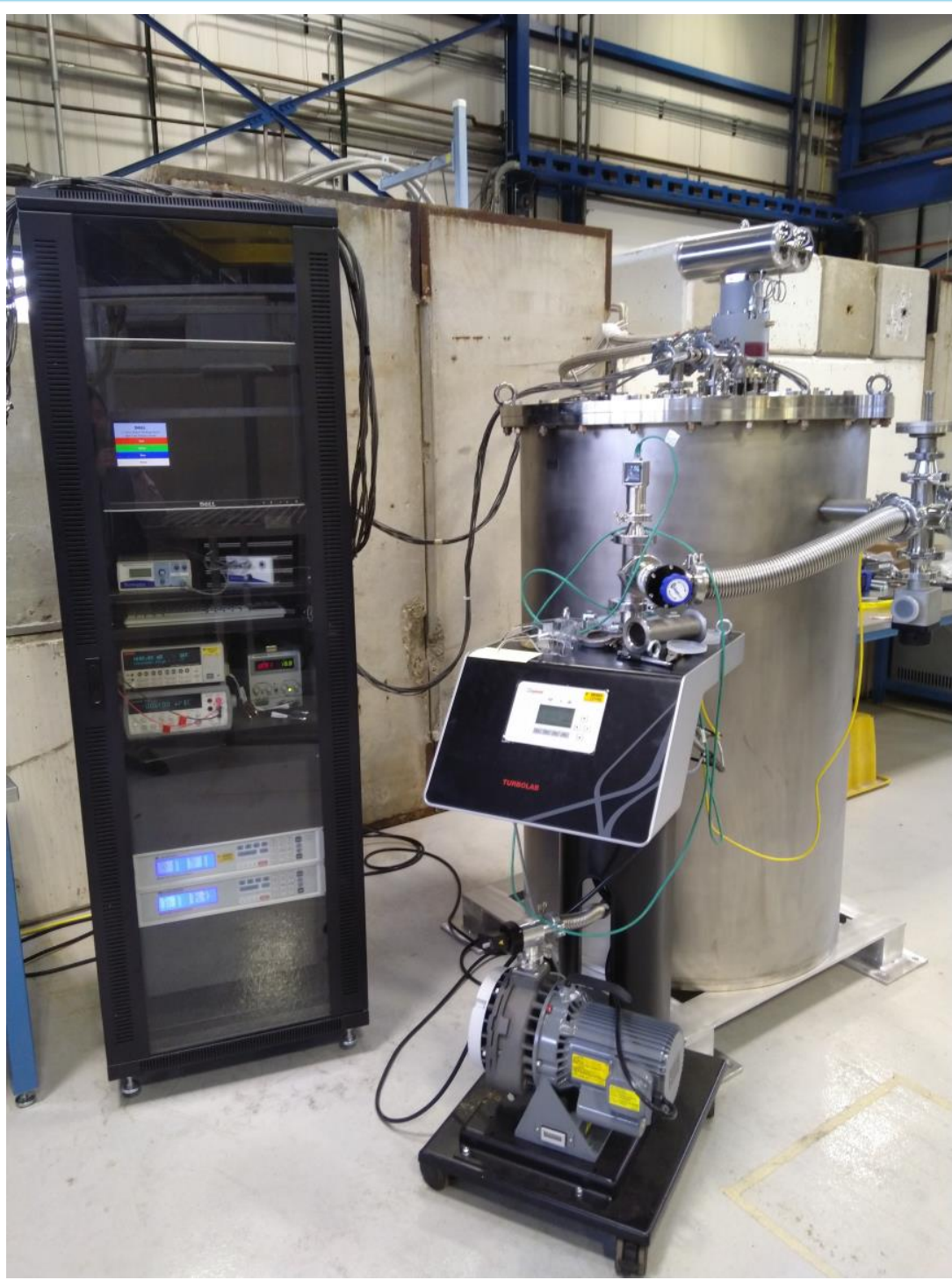

帏 Fermilab 


\section{Cool down characteristics}

The cryostat cooled to its base temperature within 24 hours

- Cryocooler stage $\mathrm{I}<30 \mathrm{~K}$, thermal shield top plate $\approx 32 \mathrm{~K}$

- Cryocooler stage II $\approx 2.95 \mathrm{~K}$

- Cavity cell $\approx 5$ - $5.8 \mathrm{~K}$ (measured at multiple locations)

A possible reason for the significant cryocooler-cavity $\Delta T$

- The estimated heat leak to cryocooler $4 \mathrm{~K}$ stage is $\approx 450 \mathrm{~mW}$, mostly coming via the RF cables

- This heat flows through the cavity body (4 $\mathrm{mm}$ thick niobium), then through the thermal link, and into the cryocooler

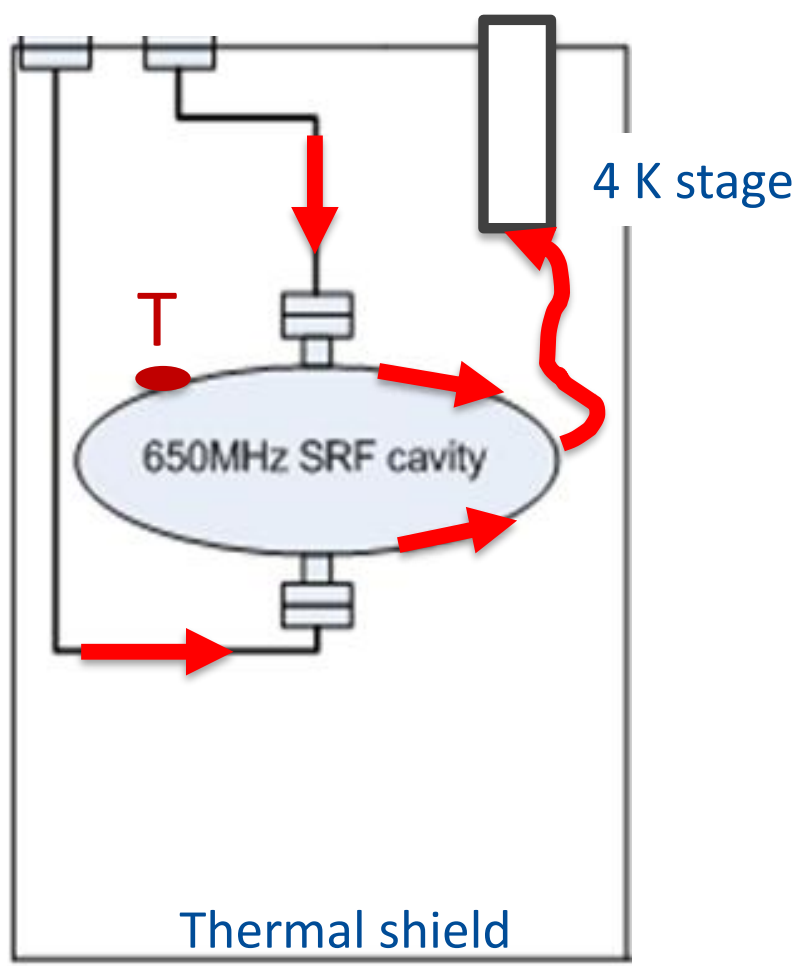




\section{First results: Accelerating gradient $>1.5 \mathrm{MV} / \mathrm{m}$}

- First measurements used a single cell, $650 \mathrm{MHz}$, niobium cavity

- Cryocooler had available $\sim 1.55 \mathrm{~W} @ 4.2 \mathrm{~K}$ after accounting for the static leaks

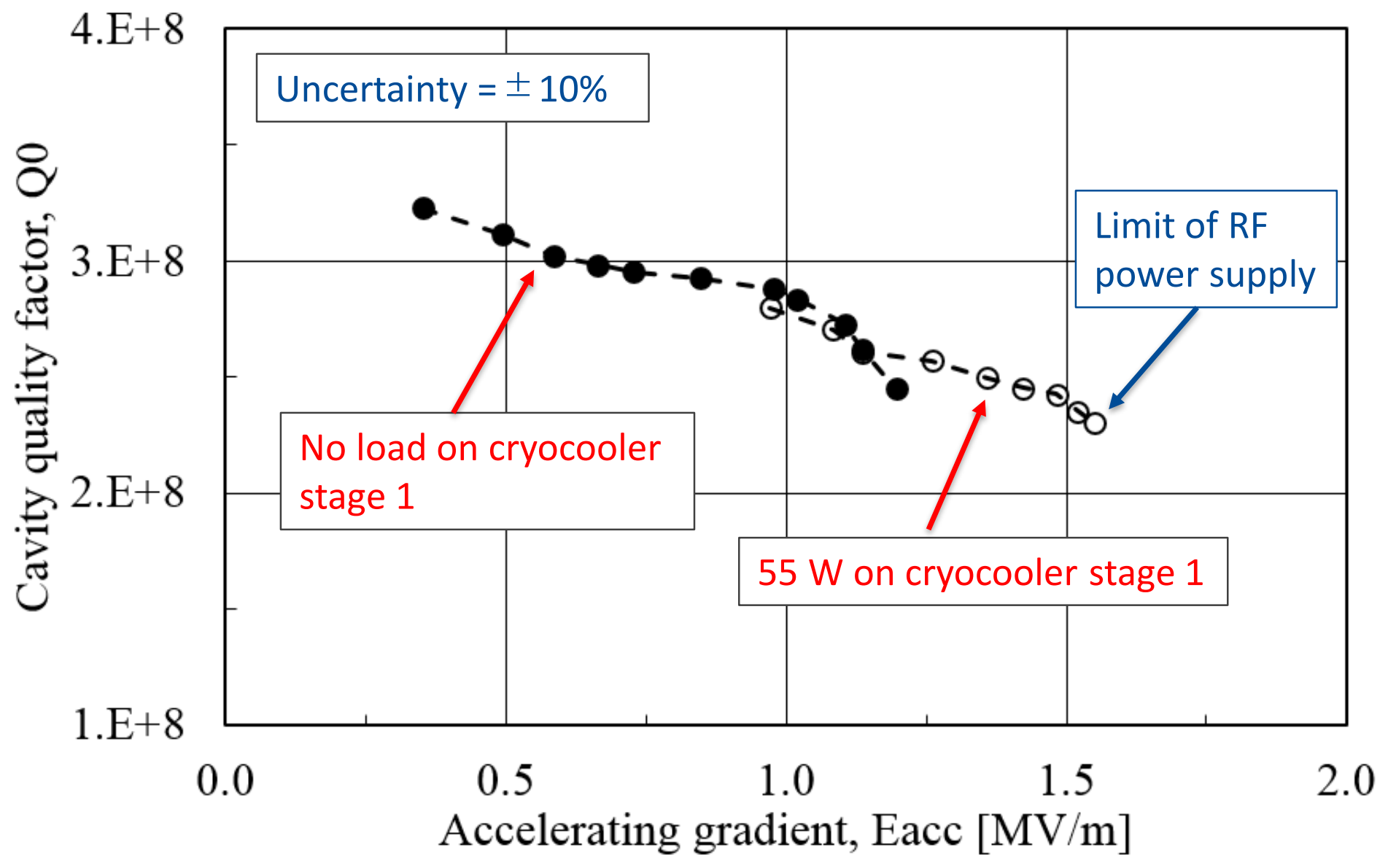




\section{Projections for a $\mathrm{Nb}_{3} \mathrm{Sn}$ coated cavity with the existing link}

Need to know the cavity RF surface and cryocooler temperatures

- $T_{\text {cavity,RF }}$ is estimated from $Q_{0}, T_{\text {cryocooler }}$ is measured

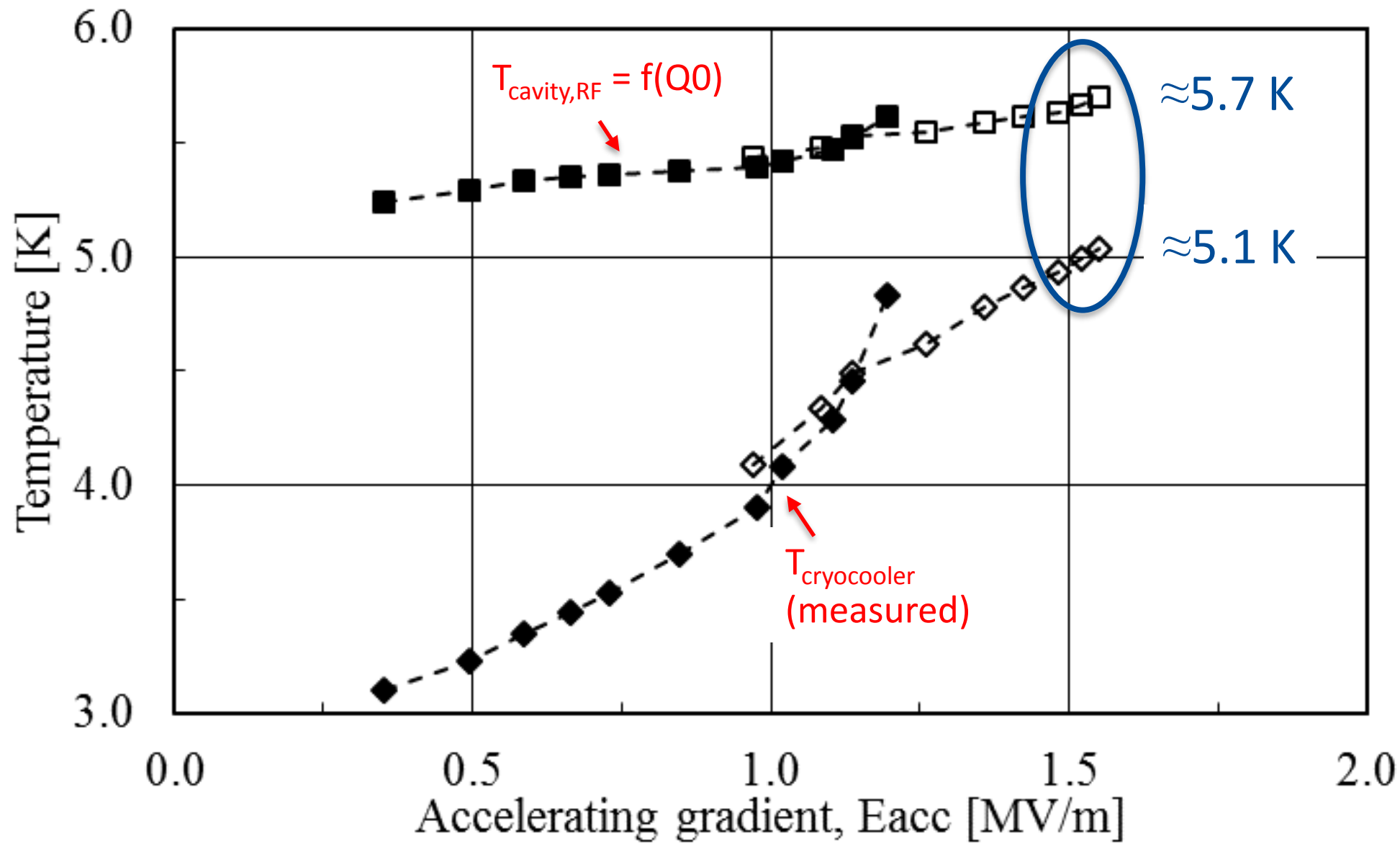

(a measure of heat dissipation in the cavity) 


\section{Projections for a $\mathrm{Nb}_{3} \mathrm{Sn}$ coated cavity with the existing link}

Assume no changes to the link

- $\mathrm{T}_{\text {cavity }, \mathrm{RF}}=5.7 \mathrm{~K}$

- $T_{\text {cryocooler }}=5.1 \mathrm{~K}$

$E_{a c c} \propto \frac{1}{\sqrt{R_{B C S}(T)+R_{\text {residual }}}}$

$\mathrm{Nb}_{3} \mathrm{Sn}<<$ Niobium $\quad \mathrm{Nb}_{3} \mathrm{Sn}$ has demonstrated (see plot) as low as $10 \mathrm{n} \Omega$

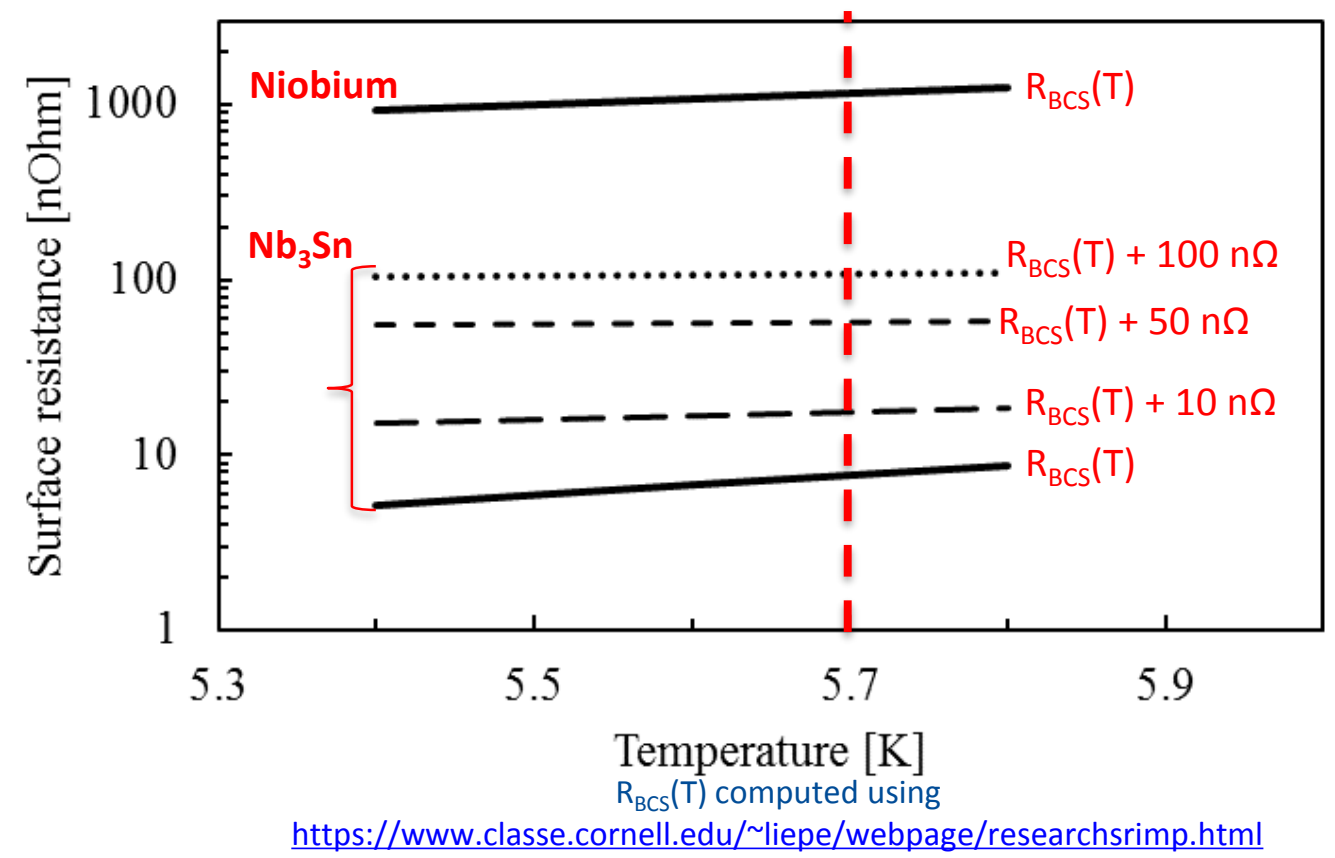

Projected $\mathrm{E}_{\text {acc }}$ for $\mathrm{Nb}_{3} \mathrm{Sn}$ with different

Surface resistance residuals

\begin{tabular}{|c|c|}
\hline \begin{tabular}{c} 
in $\mathbf{N b}_{\mathbf{3}} \mathbf{S n}[\mathbf{n} \boldsymbol{\Omega}]$ \\
\hline $20($ residual $=10)$
\end{tabular} & $\begin{array}{c}\mathbf{E}_{\text {acc }} \\
\text { conduction-cooling link }\end{array}$ \\
\hline $60($ residual $=50)$ & 11.5 \\
\hline $110($ residual $=100)$ & 6.5 \\
\hline
\end{tabular}




\section{Summary and outlook}

First ever demonstration of accelerating gradients on a cryogen-free, cryocooler conduction-cooled SRF cavity

- Niobium cavity produced $>1.5 \mathrm{MV} / \mathrm{m}$ with a $2 \mathrm{~W} @ 4.2 \mathrm{~K}$ cryocooler

- There is considerable scope for improving the thermal management in our setup

- Ongoing: mitigation of static heat leak

- $A n \mathrm{Nb}_{3} \mathrm{Sn}$ coated cavity is projected to yield $>10 \mathrm{MV} / \mathrm{m}$ accelerating gradients on our existing setup

- Tests are planned for the near future 
This presentation has been authored by Fermi Research Alliance, LLC under Contract No. DE-AC02-07CH11359 with the U.S. Department of Energy, Office of Science, Office of High Energy Physics. 


\section{Thank you.}

